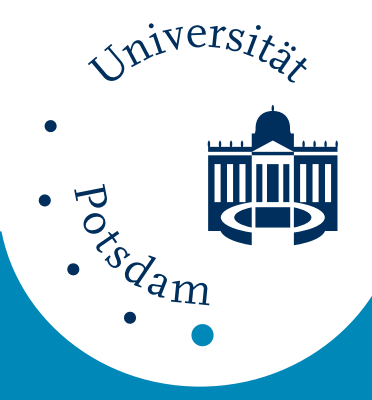

Universität Potsdam

Ayhan Demircan, Norbert Seehafer

Nonlinear square patterns in Rayleigh-Bénard convection

NLD Preprints ; 62 


\title{
Nonlinear square patterns in Rayleigh-Bénard convection
}

\author{
A. Demircan and N. Seehafer \\ Institut für Physik, Universität Potsdam, Postfach 6015 53, 14415 Potsdam, Germany
}

PACS. 47.20.Ky - Nonlinearity (including bifurcation theory).

PACS. 47.20.Bp - Buoyancy-driven instability.

PACS. 47.54.+r - Pattern selection; pattern formation.

\begin{abstract}
We numerically investigate nonlinear asymmetric square patterns in a horizontal convection layer with up-down reflection symmetry. As a novel feature we find the patterns to appear via the skewed varicose instability of rolls. The time-independent nonlinear state is generated by two unstable checkerboard (symmetric square) patterns and their nonlinear interaction. As the bouyancy forces increase the interacting modes give rise to bifurcations leading to a periodic alternation between a nonequilateral hexagonal pattern and the square pattern or to different kinds of standing oscillations.
\end{abstract}

Pattern formation occurs in a wide variety of extended physical systems and is an area of active research. One of the most frequently studied pattern-forming systems is Rayleigh-Bénard convection, i.e., bouyancy-driven convection in a fluid layer heated from below [1]. Recent experiments in Boussinesq Rayleigh-Bénard convection show unexpected patterns which appear in a range where only rolls were known to be stable. These patterns do not occur near the threshold for convection and emerge when the roll pattern disappears. Assenheimer and Steinberg [2] observed patterns consisting of domains of upflow hexagons (with rising motion in the cell center) coexisting with domains of downflow hexagons, which appear via a core instability of spirals and targets. The main feature of the observed hexagonal patterns is that the wavelength of the hexagons is significantly larger than that of rolls at the same Rayleigh number. Using an appropriate Galerkin ansatz for steady Boussinesq convection, Clever and Busse [3] calculated hexagon solutions and proved them to be stable in the regions of Rayleigh-wave number space where they were observed in the experiment. In [4] Busse and Clever showed that a similar property holds for convection flows in the form of squares. So called asymmetric squares with rising or with descending motion in the center (and descending or rising motion near the boundary) become stable at elevated Rayleigh numbers. An experimental observation indicating a tendency towards coexisting up and downflow square and hexagonal cells is also shown in [4]. In the studies [2-4] the material properties and the boundary conditions are symmetric about the midplane of the convective layer. Usually, hexagons and asymmetric squares are observed in convection lacking up-down reflection symmetry, namely in fluids with strongly temperature dependent viscosity [5] or in Bénard-Marangoni convection. They are also found in other systems, for instance among the standing wave patterns observed in the Faraday experiment, in which waves on the free surface of a fluid layer are produced by 
subjecting the layer to vertical oscillations, if a two-frequency excitation is used [6,7]; in similar experiments with magnetically driven ferrofluids already a single-frequency forcing (which, however, simultaneously excites two spatial wave numbers) can generate the patterns [8]. In [6] the observation of asymmetric squares in a two-frequency Faraday experiment with forcing frequencies in the ratio $3 / 5$ is reported. For this kind of "odd/odd" forcing the Faraday system resembles Boussinesq convection with up-down reflection symmetry in that quadratic terms in the relevant amplitude equation (valid near the primary bifurcation of the basic state) are excluded by symmetry; in the case of non-Boussinesq convection (convection with temperature-dependent viscosity) such quadratic terms are present and enable triadic wave vector interactions that can explain, for instance, the occurence of hexagonal patterns. In the case of Bénard-Marangoni convection a time-independent square pattern can be observed to evolve from a hexagonal planform [9-11]. The transition takes place via a merging of pairs of threefold vertices into single fourfold vertices (see fig. 5 below).

The qualitative behavior of these kinds of patterns is not well understood at present. To gain insight into the appearance and properties of the asymmetric square pattern we investigate a fluid layer with up-down symmetry. In our simulations the square pattern appears via the skewed varicose instability of rolls (see, e.g., [12]). It is determined by modes with two different wave numbers that are simultaneously excited and the nonlinear interaction between them. In the Faraday experiment a simultaneous excitation of two different wave numbers is reached by two-frequency forcing, and many of the patterns observed for this forcing can be explained by nonlinear interactions of modes whose wave vectors form resonant triads $[13,14]$. We find the nonlinear square pattern to exhibit interesting spatial and temporal behavior. Mode interaction gives rise to a bifurcation that leads to an oscillation consisting of a periodic alternation between a hexagonal pattern and a square pattern due to a dynamical version of the instability of a fourfold vertex, known as $T_{1}$ process [15]. This oscillatory behavior of dynamical side swapping in square convection was observed by Ondarçuhu et al. [16] in a Bénard-Marangoni convection experiment in a small aspect ratio square vessel. The pattern can also undergo bifurcations to other kinds of oscillatory solutions. So a special case of a double Hopf bifurcation with strong resonance, induced by symmetry, is observed where two kinds of standing oscillations bifurcate simultaneously.

We consider buoyancy-driven convection in a plane fluid layer of thickness $d$ heated from below. Using the Oberbeck-Boussinesq approximation, the governing system of partial differential equations reads as follows:

$$
\begin{aligned}
\frac{\partial \boldsymbol{v}}{\partial t}+(\boldsymbol{v} \cdot \nabla) \boldsymbol{v} & =-\nabla p+\mathcal{P} \triangle \boldsymbol{v}+\mathcal{P} \mathcal{R} \theta \boldsymbol{e}_{z} \\
\nabla \cdot \boldsymbol{v} & =0 \\
\frac{\partial \theta}{\partial t}+\boldsymbol{v} \cdot \nabla \theta & =v_{z}+\triangle \theta
\end{aligned}
$$

Here $\boldsymbol{v}$ is the fluid velocity and $p$ and $\theta$ represent the deviations of pressure and temperature from their values in the pure conduction state. We use Cartesian coordinates $x, y$ and $z$ with the $z$ axis in the vertical direction parallel to the gravitational force. $\boldsymbol{e}_{z}$ is the unit vector in the vertical direction. Equations (1)-(3) are given in dimensionless form where the units of length and time are $d$ and $d^{2} / \kappa$ respectively, with $\kappa$ being the thermal diffusivity. $\theta$ is measured in units of the temperature difference $\delta T$ between the lower and upper boundaries of the fluid layer. There are two dimensionless parameters, the Prandtl number $\mathcal{P}$ and the Rayleigh number $\mathcal{R}$, defined by

$$
\mathcal{P}=\frac{\nu}{\kappa}, \quad \mathcal{R}=\frac{\alpha g d^{3}}{\nu \kappa} \delta T,
$$


where $\nu$ is the kinematic viscosity, $\alpha$ the volumetric expansion coefficient and $g$ the gravitational acceleration. The Rayleigh number $\mathcal{R}$ measures the strength of the buoyancy forces. We fix $\mathcal{P}$ to a value of 6.8 and apply periodic boundary conditions with spatial period $L$ in the horizontal directions $x$ and $y$. The top and bottom planes are assumed to be impenetrable, stress-free and isothermal:

$$
\frac{\partial v_{x}}{\partial z}=\frac{\partial v_{y}}{\partial z}=v_{z}=\theta=0 \quad \text { at } z=0,1 .
$$

We wish to emphasize that the qualitative bifurcation behavior of our system is determined by its symmetry and is thus the same as for identical rigid-wall conditions at the top and bottom (though the critical Rayleigh numbers at which the bifurcations occur are different). We restrict ourselves to the case of a vanishing mean horizontal flow (corresponding to vanishing Fourier coefficients of $v_{x}$ and $v_{y}$ for wave number $\boldsymbol{k}=\mathbf{0}$ ) since such flows can be removed by a Galiliean transformation. In our numerics we used a pseudospectral method $[17,18]$ as described in [19]. The spatial resolution was 32 points in each direction, with the exception of (non-simulative) eigenvalue and eigenvector calculations, where only 16 points in each direction were used. Time integration was performed using an eighth-order Runge-Kutta scheme as described in [20].

An example of steady down-square convection is shown in fig. 1a. Results of a stability analysis are shown in fig. 2. The square pattern appears via the skewed varicose instability

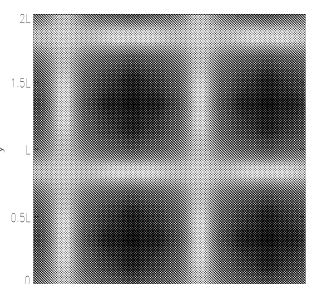

(a)

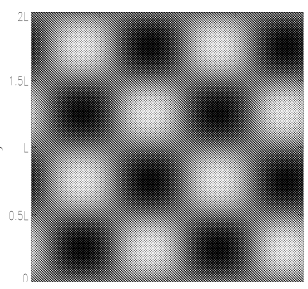

(b)

Fig. 1 - Shadowgraph images of the vertical velocity component $v_{z}$ in the horizontal midplane for $L=4$. Bright areas refer to positive values, where the motion of the fluid is upwards. (a) Asymmetric square pattern for $\mathcal{R}=2000$, (b) unstable checkerboard pattern for $\mathcal{R}=1010$.

of rolls. Most of the calculations were carried out in the aspect ratio range $3.5 \leq L \leq 5$, where one finds a pattern with just one square in the periodic box. In additional calculations for aspect ratios between 5 and 8 patterns with two square cells (lying under $45^{\circ}$ in the box) or four cells, respectively, were found (two cells for $5 \lesssim L \lesssim 7$ and four cells for $L \gtrsim 7$, where the $L$ intervals for different numbers of cells overlap at the ends). For aspect ratios smaller than about 3 the skewed varicose instability is suppressed and no squares appear. In this case the primary rolls are stable up to significantly higher Rayleigh numbers and lose then stability to traveling waves along the roll axis [19].

For $3.5 \leq L \leq 5$ and close to the onset of convection, we find two pairs of purely twodimensional, straight, stable, stationary convection rolls lying under $45^{\circ}$ in the box. When the Rayleigh number crosses the instability line specified as a dashed line in fig. 2, four equal real eigenvalues become positive and the roll pattern becomes unstable to perturbations with 


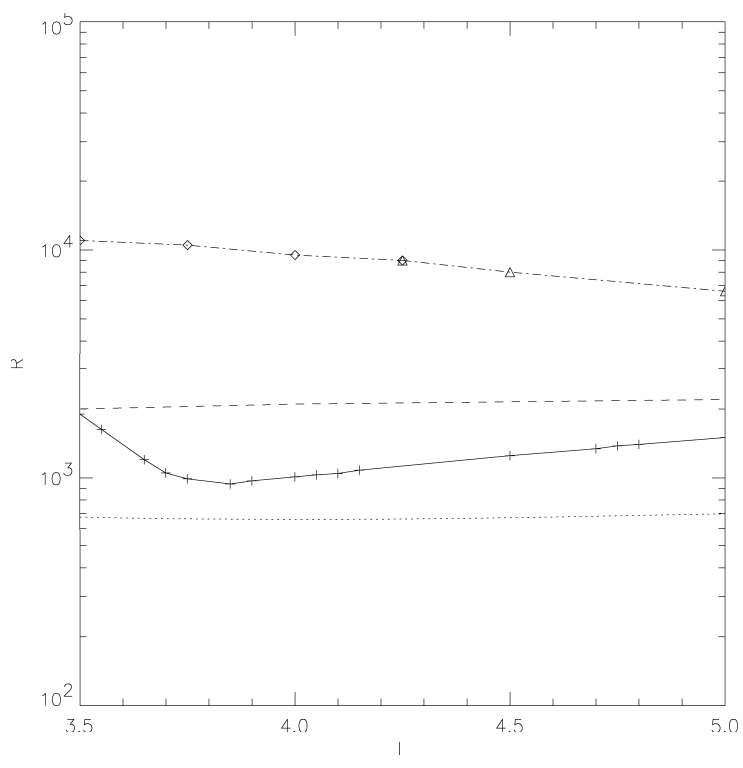

Fig. 2 - The region of stable squares in the $L$ - $\mathcal{R}$ plane. The dashed line specifies the skewed varicose instability for rolls and indicates the transition from a roll pattern to a square pattern for increasing Rayleigh number. The back transition from the squares to the rolls for decreasing $\mathcal{R}$ is shown by the solid line, with + signs marking the calculated points. The dashed-dotted line indicates instability of the squares for increasing $\mathcal{R}$, diamonds denoting a double Hopf bifurcation and triangles a single Hopf bifurcation. For reference, the linear stability boundary of the nonconvective ground state to rolls is shown by the dotted line.

the characteristic skewed varicose appearance of the disturbed pattern, caused by a periodic thickening and thinning of the convection rolls. This (transient) deformation is followed by a transition to a stable stationary square pattern in combination with a jump to a significantly higher Nusselt number (measuring the rate of heat transfer through the convection layer). The latter indicates that the stable squares do not bifurcate supercritically from the roll-solution branch. Cells with rising motion as well as cells with descending motion in the center can appear - this must be so since the system is symmetric with respect to up-down reflections.

The skewed varicose instability of the rolls can lead to different branches of asymmetric squares which seem to bifurcate simultaneously. In simulations starting from a superposition of the unperturbed rolls with a small perturbation one always observes a transient skewed varicose pattern, but, depending on the perturbation added to the rolls, the final square solution may or may not be symmetric with respect to the dihedral group $D_{4}$ of all rotations and reflections of a square in a plane which leave the square invariant. In the following we report results for the $D_{4}$ symmetric branch. When looking at the spectrum of the excited Fourier modes of solutions on this branch, one sees that to lowest order they can be represented by

$$
A\left(e^{i \boldsymbol{k}_{1} \boldsymbol{x}}+e^{i \boldsymbol{k}_{2} \boldsymbol{x}}\right)+B\left(e^{i\left(\boldsymbol{k}_{1}+\boldsymbol{k}_{2}\right) \boldsymbol{x}}+e^{i\left(\boldsymbol{k}_{1}-\boldsymbol{k}_{2}\right) \boldsymbol{x}}\right)+\text { c.c. }
$$

where $\boldsymbol{k}_{1}$ and $\boldsymbol{k}_{2}$ are horizontal wave numbers, $\boldsymbol{k}_{1} \perp \boldsymbol{k}_{2}$, and $\left|\boldsymbol{k}_{1}\right|=\left|\boldsymbol{k}_{2}\right|=k$ is the dominating wave number of the square pattern ( $2 \pi / k$ is the side length of the asymmetric squares). The coefficients $A$ and $B$ are of the same order of magnitude, while the coefficients of all other 
Fourier modes are smaller by at least one order of magnitude. Each of the two summands in eq. (6) describes a checkerboard pattern as shown in fig. 1b (with either upward or downward motion over a whole square), the summand with the coefficient $A$ one with the dominating wavelength and the summand with the coefficient $B$ another one, rotated by an angle of $\pi / 4$, with a side length smaller by the factor $1 / \sqrt{2}$. Both checkerboard patterns are unstable, in contrast to their superposition, the asymmetric square pattern. The second wave number, $q=\left|\boldsymbol{k}_{1}+\boldsymbol{k}_{2}\right|=\sqrt{2} k$, is the wave number of the roll solution that becomes unstable to the skewed varicose instability; for Rayleigh numbers above the critical one for the instability this roll state exists as an unstable solution. Rolls with the smaller wave number $k$ are stable in the region where we observe stable asymmetric squares. The periodic boundary conditions with fixed aspect ratio admit only discrete values of the angle between the rolls and (say) the $y$ axis and of the wave number of the rolls - since even integer numbers of rolls have to cross the sides of the periodic box (cf. [21]). $q$ and $k$ are two admitted wave numbers that are in resonance through the condition $q=\left|\boldsymbol{k}_{1} \pm \boldsymbol{k}_{2}\right|,\left|\boldsymbol{k}_{1}\right|=\left|\boldsymbol{k}_{2}\right|=k$. As a consequence of the discreteness, they do not vary with $\mathcal{R}$ but are fixed. Assenheimer and Steinberg [2] experimentally observed coexisting rolls and hexagons with a wave number ratio of 1.2 to 1.3 between rolls and hexagons. The aspect ratio of the experimentally studied layer was large and rolls and hexagons were seen in the same pattern. In our calculations, for small aspect ratio, pure roll or square patterns are found as time-asymtotic states.

The nonlinear interaction between the two ckeckerboard solutions (with wave numbers $k$ and $q$, respectively) determines the stationary state and leads to a square pattern with upflow (downflow) motion in the centers and downflow (upflow) boundaries. This is contained in the Galerkin ansatz for a steady three-dimensional solution in [4]. An ansatz like eq. (6) was also used by Proctor and Matthews [22] in a study of square cells in non-Boussinesq convection near onset, while Dawes [23] analysed a $1: \sqrt{2}$ resonance between an oscillatory and a steady mode in magnetoconvection.

The stability boundary of the asymmetric squares towards lower values of $\mathcal{R}$, where there is a back transition to stable rolls of wave number $q$, is obtained by following the path backwards and calculating the eigenvalues and eigenvectors of the linear stability problem close to the Rayleigh number where the squares appeared. The critical unstable eigenvector (corresponding to a single real eigenvalue) is found to contain only $q$-dependent modes. Furthermore, during the transition from asymmetric squares to rolls a transient checkerboard pattern with the wavelength $q$ of the rolls can be observed (fig. 1b). As is seen in fig. 2, the stable squares can be traced to Rayleigh numbers below the critical one for the skewed varicose instability of the rolls. There is a bistable region (between the solid and the dashed line in fig. 2) where both stable rolls and stable asymmetric squares exist (i.e., depending on the initial conditions, the system can converge to a roll pattern with wave number $q$ or to a square pattern). This hysteretic behavior indicates that the bifurcation of the squares from the rolls is subcritical.

We have also traced the square pattern solution for increasing values of the Rayleigh number and have observed two different kinds of Hopf bifurcation, depending on the aspect ratio $L$. These bifurcations are apparently supercritical since neither a jump in the Nusselt number nor hysteresis are observed. For smaller $L$ two identical pairs of complex conjugate eigenvalues pass the imaginary axis (denoted by diamonds in fig. 2). This is a special case of a double Hopf bifurcation with strong resonance, induced by the $D_{4}$ symmetry [24,25]. Several solutions can bifurcate simulteneously here. We find two stable solutions that correspond to standing oscillations. One of them, shown in fig. 3, appears like the superposition of two standing waves, one with oscillations in the $x$ direction and the other with oscillations in the $y$ direction, the vertex points of the square pattern being located at the nodes of the standing waves. The time period in our time units (thermal diffusion times) is $\tau=0.11$. The spatial 


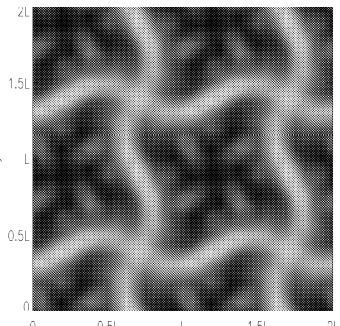

(a)

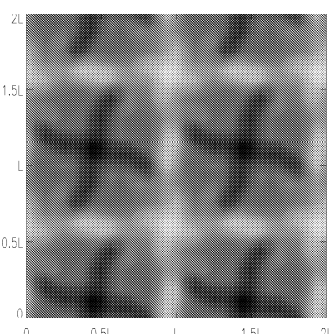

(b)

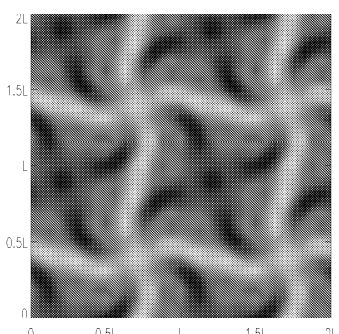

(c)

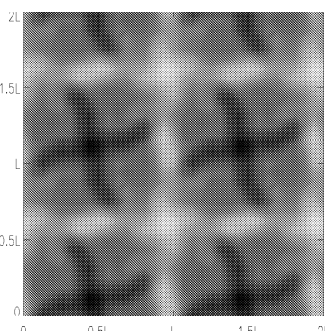

(d)

Fig. 3 - Successive shadowgraph images of the vertical velocity component $v_{z}$ in the horizontal midplane for a time-periodic state at Rayleigh number $\mathcal{R}=8000$ in a cell of aspect ratio $L=4.1$. Snapshots are taken at $t=0(\mathrm{a}), t=\tau / 4(\mathrm{~b}), t=\tau / 2$ (c) and $t=3 \tau / 4(\mathrm{~d})$, where $\tau$ is the time period.

symmetry $D_{4}$ is broken down to $Z_{4}$ (the cyclic group). For the second standing oscillation, shown in fig. 4 , the $D_{4}$ symmetry is broken down to a reflection symmetry with respect

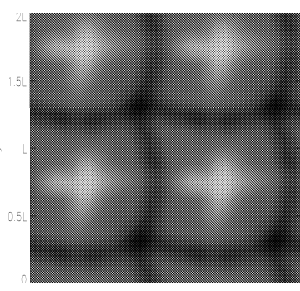

(a)

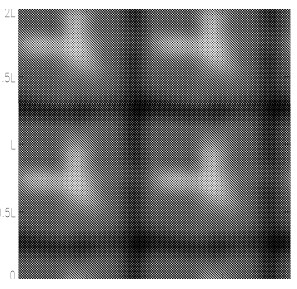

(b)

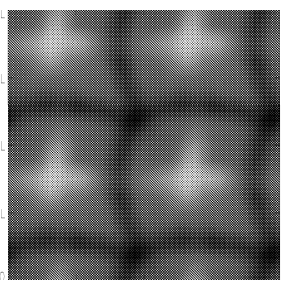

(c)

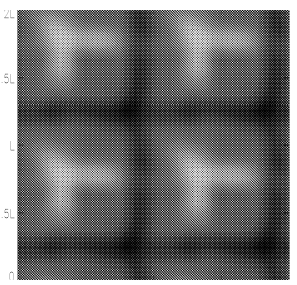

(d)

Fig. 4 - Successive shadowgraph images of the vertical velocity component $v_{z}$ in the horizontal midplane for a time-periodic state at Rayleigh number $\mathcal{R}=10500$ in a cell of aspect ratio $L=3.75$. Snapshots are taken at $t=0$ (a), $t=\tau / 4$ (b), $t=\tau / 2$ (c) and $t=3 \tau / 4$ (d), where $\tau$ is the time period.

to a diagonal line and the four vertex points of the square pattern oscillate with maximum amplitude and in phase parallel to the invariant diagonal.

A further kind of oscillation results from a single Hopf bifurcation, indicated by triangles in fig. 2. It consists of a periodic alternation between two nonequilateral hexagonal patterns, seen in fig. 5a and fig. 5c. These are at right angles to each other and during the transition between them the square pattern is passed through. The topology and dynamics of the whole pattern transformation can be described by a dynamical version of an elementary topological transformation in two dimensions, the $T_{1}$ process or side swapping. The spatial symmetry is broken from $D_{4}$ to $D_{2}$ (the dihedral group generated by two reflections). The oscillation period, determined by the imaginary part of the critical eigenvalues, is $\tau=0.23$ in our time units. This oscillatory behavior of dynamical side swapping in square convection was observed 


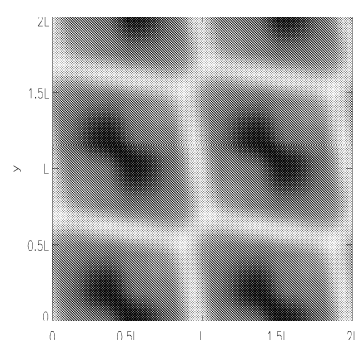

(a)

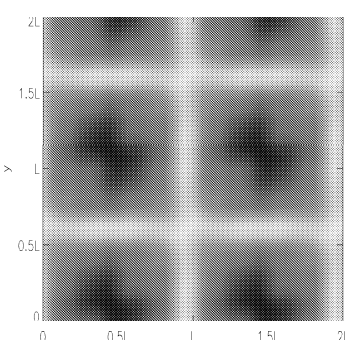

(b)

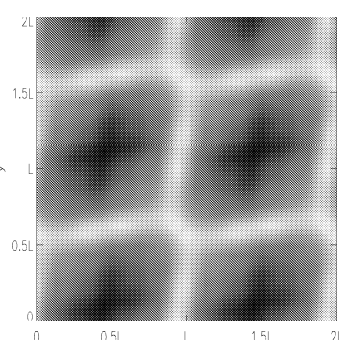

(c)

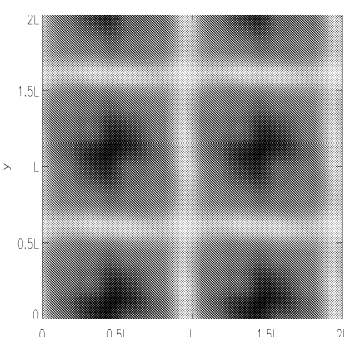

(d)

Fig. 5 - Successive shadowgraph images of the vertical velocity component $v_{z}$ in the horizontal midplane for a time-periodic state at Rayleigh number $\mathcal{R}=5800$ in a cell of aspect ratio $L=4.5$. Snapshots are taken at $t=0(\mathrm{a}), t=\tau / 4(\mathrm{~b}), t=\tau / 2(\mathrm{c})$ and $t=3 \tau / 4(\mathrm{~d})$, where $\tau$ is the time period.

by Ondarçuhu et al. [16] in a Bénard-Marangoni convective experiment in a small aspect ratio square vessel $(L=4.46)$. They observed a sequence of qualitative changes in a convection pattern consisting of four square cells, which are determined by the dynamics of one vertex. In [26] this behavior is described by the nonlinear interaction between different critical modes whose linear superposition generates the experimentally observed pattern. Transformation from a hexagonal pattern into a square pattern via a merging of cell knots has been observed in [9], [11] and [27]. These experiments were done for Bénard-Marangoni convection, but they exhibit instabilities for patterns with a square symmetry in general. The symmetry determines which kinds of patterns can appear through instabilities and bifurcations. Once the asymmetric squares have appeared in our Boussinesq system, the up-down reflection symmetry is broken and the behavior can be expected to resemble that of asymmetric squares in BénardMarangoni convection.

In conclusion, we have numerically investigated the appearance and properties of nonlinear asymmetric square patterns in Boussinesq Rayleigh-Bénard convection with up-down symmetry. The squares were found to appear via the skewed varicose instability of rolls, without controlled initial conditions and without any special ansatz. They result from the nonlinear interaction of two unstable checkerboard (symmetric square) patterns with a sidelength ratio of $1: \sqrt{2}$. A jump in the Nusselt number indicates that stable squares do not bifurcate supercritically from the primary roll-solution branch. Hysteresis of the transition points to a subcritical bifurcation. Towards higher Rayleigh numbers the square pattern loses stability to different kinds of oscillations. To a large part the qualitative behavior of the calculated solutions has been seen in experiments.

\section{REFERENCES}

[1] Cross M. C. and Hohenberg P. C., Rev. Mod. Phys., 65 (1993) 851.

[2] Assenheimer M. and Steinberg V., Phys. Rev. Lett., 76 (1996) 756.

[3] Clever R. M. and Busse F. H., Phys. Rev. E, 53 (1996) R2037.

[4] Busse F. H. and Clever R. M., Phys. Rev. Lett., 81 (1998) 341.

[5] Oliver D. S. and Booker J. R., Geophys. Astrophys. Fluid Dyn., 27 (1983) 73.

[6] Edwards W. S. and Fauve S., J. Fluid Mech., 178 (1994) 123. 
[7] Arbell H. and Fineberg J., Phys. Rev. Lett., 81 (1998) 4384.

[8] Pi H.-J., Park S., Lee J., and Lee K. J., Phys. Rev. Lett., 84 (2000) 5316.

[9] Nitschke K. and Thess A., Phys. Rev. E, 52 (1995) R5772.

[10] M. Bestehorn, Phys. Rev. Lett., 76 (1996) 46.

[11] Schatz M. F., VanHook S. J., McCormick W. D., Swift J. B. and Swinney H. L., Phys. Fluids, 11 (1999) 2577.

[12] Busse F. H. and Clever R. M., J. Fluid Mech., 91 (1979) 319.

[13] Silber M. and Skeldon A. C., Phys. Rev. E, 59 (1999) 5446.

[14] Arbell H. and Fineberg J., Phys. Rev. Lett., 84 (2000) 654.

[15] Rivier N., J. Phys.: Condens. Matter, 4 (1992) 931.

[16] Ondarçuhu T., Mindlin G. B., Mancini H. L. and Pérez-García C., Phys. Rev. Lett., 70 (1993) 3892.

[17] Canuto C., Hussaini M. Y., Quarteroni A. and Zang T. A., Spectral Methods in Fluid Dynamics (Springer, Berlin) 1988.

[18] Press W. H., Flannery B. P., Teukolsky S. A. and Vetterling W. T., Numerical Recipes, The Art of Scientific Computing, FORTRAN Version (Cambridge University Press, Cambridge, England) 1989.

[19] Scheel S. and Seehafer N., Phys. Rev. E, 56 (1997) 5511.

[20] Hairer E., Nørsett S. P. and Wanner G., Solving Ordinary Differential Equations I (Springer, Berlin) 1993.

[21] Demircan A., Scheel S. and Seehafer N., Eur. Phys. J. B, 13 (2000) 765.

[22] Proctor M. R. E. and Matthews P. C., Physica D, 97 (1996) 229.

[23] Dawes J. H. P., Physica D, 139 (2000) 109.

[24] Golubitsky M., Stewart I. and Schaeffer D. G., Singularities and Groups in Bifurcation Theory (Springer, New York) 1988, Vol. II.

[25] Swift J. W., Nonlinearity, 1 (1988) 333.

[26] Krmpotić D., Mindlin G. B. and Pérez-García C., Phys. Rev. E, 54 (1996) 3609.

[27] Eckert K., Bestehorn M. and Thess A., J. Fluid Mech., 356 (1998) 155. 